
\title{
and Bone Mineral Density in Korean Adolescents: The Fifth Korea National Health and Nutrition Examination Survey (KNHANES V-1), 2010
}

\author{
Hee-Cheol Jeon, Kayoung Lee*, Jinseung Kim*, Tae-Jin Park, Dae-Won Kang, \\ Da-Jung Park
}

Department of Family Medicine, Busan Paik Hospital, College of Medicine, Inje University, Busan, Korea

Background: The relationships of total and regional body fat percent with bone mineral density (BMD) in Korean adolescents were examined using the Fifth Korea National Health and Nutrition Examination Survey (KNHANES V-1), 2010.

Methods: Body fat percent at whole body (WBFP), trunk (TBFP), and extremities (both upper and lower extremities fat mass/body weight, EBFP), ratio of trunk fat mass to extremities fat mass (TEFR), and BMD at whole body, total femur, and lumbar spine were measured by dual energy X-ray absorptiometry in a population-based sample of 433 boys and 362 girls, aged 12 to 18 years. The analyses were conducted using linear regression analysis with complex sampling design.

Results: After adjusting for confounders such as age, height, weight, serum 25-(OH) vitamin D concentration, energy intake, calcium intake, physical activity, and menarche status for girls, WBFP, TBFP, and EBFP were inversely associated with whole and regional BMD in both sexes $(\mathrm{P}<0.05)$. TEFR was positively associated with whole and regional BMD in boys after adjusting for confounders, while it was negatively associated in girls $(\mathrm{P}<0.05)$. However, the associations were non-significant when bone mass-free lean mass was adjusted instead of bodyweight except for a positive association between TEFR and BMD in boys.

Conclusion: In Korean adolescents, total and regional body fat percent is not independently associated with BMD after adjusting for bone mass-free lean mass but higher fat in trunk as compared to extremities may be protective for BMD in boys.

Keywords: Adipose Tissue; Bone Density; Adolescent

Received: August 16, 2012, Accepted: October 7, 2014

${ }^{*}$ Corresponding Author: Kayoung Lee

Tel: +82-51-890-6229, Fax: +82-51-894-7554

INTRODUCTION

E-mail: fmlky@inje.ac.kr

${ }^{*}$ Corresponding author: Jinseung Kim

Tel: +82-51-890-6229, Fax: +82-51-894-7554

E-mail: jinseungkim@hanmail.net

Korean Journal of Family Medicine

Copyright (C) 2014 The Korean Academy of Family Medicine

() This is an open-access article distributed under the terms of the Creative Commons Attribution Non-Commercial License (http://creativecommons.org/licenses/by-nc/3.0) which permits unrestricted noncommercial use, distribution, and reproduction in any medium, provided the original work is properly cited.

Osteoporosis is a most common metabolic disease of bone and approximately $40 \%$ of postmenstrual women met the criteria of osteoporosis. ${ }^{1)}$ Osteoporosis is defined as the bone disease with low bone strength and high risk for fracture by The National Institutes of Health Consensus Development Panel on Osteoporosis Prevention, Diagnosis, and Therapy. ${ }^{2)}$ Major risk factors of osteoporosis is low bone mass in adolescence and rapid bone loss by aging and menopause. Therefore, maximizing 
bone mass in adolescence is important to prevent osteoporosis. ${ }^{3)}$ Measurement of bone mineral density (BMD) is a useful methods to evaluate osteoporosis status. ${ }^{4,5)}$ In previous studies for adults, body fat mass and lean body mass have been related to BMD. In these studies, lean body mass has been positively associated with $\mathrm{BMD}$ regardless of sex and age, while the association between body fat mass and BMD has been inconsistent according to the menopausal status ${ }^{6,7)}$ and age groups. ${ }^{8-10)}$

On the other hand, studies in adolescents have shown inconsistent results about gender difference in the relationships between body fat mass and BMD. ${ }^{11,12)}$ In addition, studies for obese adolescents reported an inverse association between abdominal fat and $\mathrm{BMD}^{13,14)}$ and a recent study about the relationship between regional $\mathrm{BMD}$ and body fat percent resulted in age and sex-specific relationships in adolescents of the Korea National Health and Nutrition Examination Survey (KNHANES) in 2009. However, that study did not examine the associations with lean body mass after taken into account of bone mass-free lean body mass. ${ }^{15)}$ Therefore, it is not clear whether the associations between body fat percent and BMD would be different by regions and independent from lean body mass free from bone mass.

The present study aimed to evaluate the relationships between BMD and body fat percent of whole body, trunk, and extremities in adolescents after adjusting for body weight or bone mass-free lean mass using the data of KNHANES in 2010.

\section{METHODS}

\section{Subjects}

The subjects of this survey included 4,115 males and 4,843 females who were over 1 year of age in 3,840 houses and participated in the KNHANES V-1 in 2010. In the subjects who were sampled using a rolling sampling design method based on the 2008 Market Price of an Apartment and 2009 Population Data of Statistical Yearbooks, ${ }^{16)} 433$ males and 362 females at the ages between 12 to 18 years were included for the analyses.

\section{Study Variables}

We used data of BMD, body composition, height, weight, serum 25-hydroxy vitamin $\mathrm{D}$ concentration, physical activity, daily energy and calcium intake, and menstrual status for girls from the KNHANES V-1. BMD $\left(\mathrm{g} / \mathrm{cm}^{2}\right)$ and body compositions were measured using a standardized method by dual energy X-ray absorptiometry (DEXA; DISCOVERY-W fan-beam densitometer Hologic Inc., Bedford, MA, USA). ${ }^{17)}$ The body composition measurements were used to derived bone massfree lean mass of whole body that was calculated by lean mass of whole body minus bone mass, whole body fat percent (WBFP), trunk fat percent (TBFP), extremities fat percent (EBFP) that was calculated by percentage of sum of both upper and lower extremities fat mass divided by weight, and ratio of trunk fat mass to extremities fat mass (TEFR). In the BMD data, we used BMD of whole body, lumbar spine bone (LSBMD), and tibiofemoral regions.

Serum 25-hydroxyl vitamin D concentration was assayed with a radioimmunoassay kit (1470 WIZARD gamma-Counter; Perkin Elmer, Turku, Finland). Physical activity was measured by self-reported questionnaires that assessed frequency of moderate and high intensity physical activity per week and walking time per day. We calculated MET-min (metabolic equivalentminutes) per week by using a physical activity calculating method of International Physical Activity Questionnaire. ${ }^{18)}$ Energy and calcium intake was assessed using a single 24-hour recall method. The menstrual history for girls was assessed using a questionnaire. $^{16)}$

\section{Statistical Analysis}

As the analyses were conducted using the complex sample design analyses in each sex. The descriptive analyses were conducted for study variables. Linear regression analysis was applied for the relationships between body fat percent and BMD after adjusting for height, weight, serum $25-\mathrm{OH}$ vitamin $\mathrm{D}$ concentration, energy and calcium intake, and physical activity, and menstrual status for girls. In this analysis, the adjustment of body weight was also replaced by bone mass-free lean mass of whole body. All the analyses were conducted using the IBM SPSS ver. 19.0 (IBM Co., Armonk, NY, USA).

\section{RESULTS}

Table 1 shows distribution of study variables in boys and girls. 
Table 1. The characteristics of boys and girls at the ages of 12 to 18 years

\begin{tabular}{|c|c|c|c|c|}
\hline \multirow{2}{*}{ Characteristic } & \multicolumn{2}{|c|}{ Boys } & \multicolumn{2}{|c|}{ Girls } \\
\hline & Mean & SE & Mean & SE \\
\hline Age (y) & 15.17 & 0.13 & 15.04 & 0.13 \\
\hline Weight (kg) & 60.71 & 0.83 & 53.27 & 0.72 \\
\hline Height $(\mathrm{cm})$ & 169.46 & 0.57 & 159.82 & 0.37 \\
\hline Bone mass-free lean mass of whole body (kg) & 44.09 & 0.52 & 33.3 & 0.29 \\
\hline Whole body fat percent & 22.19 & 0.48 & 32.85 & 0.42 \\
\hline Trunk fat percent & 20.92 & 0.53 & 30.09 & 0.55 \\
\hline Extremities fat percent & 11.01 & 0.26 & 17.11 & 0.17 \\
\hline Trunk fat mass (kg) & 5.96 & 0.23 & 7.67 & 0.29 \\
\hline Extremities fat mass (kg) & 6.84 & 0.22 & 9.26 & 0.2 \\
\hline Ratio of trunk fat mass to extremities fat mass & 0.85 & 0.01 & 0.8 & 0.01 \\
\hline Whole body BMD $\left(\mathrm{g} / \mathrm{cm}^{2}\right)$ & 1.04 & 0.01 & 1.02 & 0.01 \\
\hline Total femur BMD (g/cm²) & 0.91 & 0.01 & 0.86 & 0.01 \\
\hline Lumbar spine $B M D\left(g / \mathrm{cm}^{2}\right)$ & 0.84 & 0.01 & 0.89 & 0.01 \\
\hline Physical activity (METs · min/wk) & 34,063 & 3,919 & 43,350 & 2,713 \\
\hline Energy intake (kcal/d) & 2,554 & 74 & 1,901 & 57 \\
\hline Calcium intake (mg/d) & 574 & 24 & 432 & 20 \\
\hline Serum $25-\mathrm{OH}$ vitamin $\mathrm{D}$ & 16.6 & 0.38 & 15.4 & 0.39 \\
\hline
\end{tabular}

Analyzed using complex sampling design.

SE: standard error, BMD: bone mineral density, METs · min: metabolic equivalent-minutes.

The WBFP, TBFP, and EBFP were higher in girls as compared with boys, while TEFR was higher in boys as compared with girls. The BMD of whole body and total femur in boys were higher as compared with girls, while LSBMD was higher in girls.

Table 2 and Table 3 present the relationships between $\mathrm{BMD}$ and body fat percent in boys and girls, respectively. When the relationships were adjusted for body weight and other confounding variables, there were inverse relationships between body fat percent and BMD in both genders. In contrast, the relationships between TEFR and BMD were positive in boys, while there were inverse relationships in girls. On the other hand, in the models adjusted for bone mass-free lean mass of whole body instead of body weight, the relationships of body fat percent and TEFR with BMD were not more significant in girls, while the relationships between TEFR and BMD were still significant in boys.

\section{DISCUSSION}

Osteoporosis, a common metabolic bone disease related with aging and menopause, is an important burden of public health. Therefore, achievement of optimal bone mass in adolescents is critical for prevention of osteoporosis in later life. Bone mass reaches its peak at 18 to 25 years of age and genetic and nongenetic factors such as nutritional status, hormone, and physical activity in adolescence influence on the process of bone mass formation. ${ }^{19)}$ Among the nutritional factors, the relationships between body fat mass and BMD among adolescents have been inconsistent between studies. $^{11,12,15)}$

In the present study, we observed inverse associations between body fat percent of whole body, trunk, and extremities and $\mathrm{BMD}$ in boys and girls after adjusting for age, weight, height, serum 25-hydroxy vitamin D concentration, intake of energy and calcium, physical activity, and menstrual status for girls. 
Table 2. The relationships between body fat percent and BMD in boys at the ages of 12 to 18 years

\begin{tabular}{|c|c|c|c|c|}
\hline Variable & Category & $\begin{array}{l}\text { Whole body BMD } \\
\left(\mathrm{g} / \mathrm{cm}^{2}\right)\end{array}$ & $\begin{array}{c}\text { Total femur BMD } \\
\left(\mathrm{g} / \mathrm{cm}^{2}\right)\end{array}$ & $\begin{array}{c}\text { Lumbar spine BMD } \\
\left(\mathrm{g} / \mathrm{cm}^{2}\right)\end{array}$ \\
\hline \multirow[t]{3}{*}{ Whole body fat percent } & Age-adjusted & 0.00005 & 0.001 & 0.001 \\
\hline & Full-adjusted I $I^{\dagger}$ & -0.006 & $-0.009^{* *}$ & $-0.006^{* *}$ \\
\hline & Full-adjusted $\mathrm{II}^{*}$ & -0.001 & -0.001 & -0.001 \\
\hline \multirow[t]{3}{*}{ Trunk fat percent } & Age-adjusted & 0.001 & $0.002^{*}$ & 0.002 \\
\hline & Full-adjusted $^{\dagger}$ & $-0.006^{* *}$ & $-0.007^{* *}$ & $-0.005^{* *}$ \\
\hline & Full-adjusted $\mathrm{II}^{\dagger}$ & -0.001 & -0.001 & -0.00008 \\
\hline \multirow[t]{3}{*}{ Extremities fat percent } & Age-adjusted & -0.002 & 0.000 & 0.000 \\
\hline & Full-adjusted $^{\dagger}$ & $-0.012^{* *}$ & $-0.016^{* *}$ & $-0.012^{* *}$ \\
\hline & Full-adjusted $\mathrm{II}^{\dagger}$ & -0.004 & -0.003 & -0.002 \\
\hline \multirow[t]{3}{*}{ Ratio of trunk fat mass to extremities fat mass } & Age-adjusted & $0.200^{* *}$ & $0.264^{* *}$ & $0.272^{* *}$ \\
\hline & Full-adjusted $^{\dagger}$ & $0.127^{*}$ & $0.104^{*}$ & $0.172^{* *}$ \\
\hline & Full-adjusted $\mathrm{II}^{*}$ & $0.109^{*}$ & $0.085^{*}$ & $0.148^{* *}$ \\
\hline
\end{tabular}

Values are regression coefficient.

BMD: bone mineral density, METs · min: metabolic equivalent-minutes.

${ }^{*} \mathrm{P}<0.05 .{ }^{* *} \mathrm{P}<0.01$. ${ }^{\dagger}$ Adjusted for age, height, weight, serum 25-OH vitamin $\mathrm{D}$, physical activity (METs · min/wk), energy intake, and calcium intake using linear regression analysis with complex sampling design. ${ }^{*}$ Adjusted for age, height, bone mass-free lean mass of whole body, serum 25-OH vitamin D, physical activity (METs · min/wk), energy intake, and calcium intake using linear regression analysis with complex sampling design.

Table 3. The relationships between body fat percent and bone mineral density in girls at the ages of 12 to 18 years

\begin{tabular}{|c|c|c|c|c|}
\hline Variable & Category & $\begin{array}{l}\text { Whole body BMD } \\
\left(\mathrm{g} / \mathrm{cm}^{2}\right)\end{array}$ & $\begin{array}{c}\text { Total femur BMD } \\
\qquad\left(\mathrm{g} / \mathrm{cm}^{2}\right)\end{array}$ & $\begin{array}{c}\text { Lumbar spine BMD } \\
\left(\mathrm{g} / \mathrm{cm}^{2}\right)\end{array}$ \\
\hline \multirow[t]{3}{*}{ Whole body fat percent } & Age-adjusted & 0.001 & $0.003^{*}$ & $0.005^{* *}$ \\
\hline & 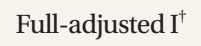 & $-0.009^{* *}$ & $-0.009^{* *}$ & $-0.007^{* *}$ \\
\hline & Full-adjusted $\mathrm{II}^{\dagger}$ & -0.002 & -0.002 & -0.0005 \\
\hline \multirow[t]{3}{*}{ Trunk fat percent } & Age-adjusted & 0.001 & $0.003^{* *}$ & $0.004^{* *}$ \\
\hline & Full-adjusted $^{\dagger}$ & $-0.007^{* *}$ & $-0.008^{* *}$ & $-0.006^{* *}$ \\
\hline & Full-adjusted $\mathrm{II}^{\dagger}$ & -0.002 & -0.001 & -0.0002 \\
\hline \multirow[t]{3}{*}{ Extremities fat percent } & Age-adjusted & 0.001 & 0.003 & 0.005 \\
\hline & Full-adjusted $^{\dagger}$ & $-0.012^{* *}$ & $-0.012^{* *}$ & $-0.011^{* *}$ \\
\hline & Full-adjusted II ${ }^{+}$ & -0.004 & -0.003 & -0.001 \\
\hline \multirow[t]{3}{*}{ Ratio of trunk fat mass to extremities fat mass } & Age-adjusted & 0.069 & $0.151^{* *}$ & $0.184^{* *}$ \\
\hline & Full-adjusted $^{\dagger}$ & $-0.123^{*}$ & -0.115 & $-0.059^{*}$ \\
\hline & Full-adjusted II & -0.053 & -0.050 & 0.017 \\
\hline
\end{tabular}

Values are regression coefficient.

BMD: bone mineral density, METs · min: metabolic equivalent-minutes.

${ }^{*} \mathrm{P}<0.05 .{ }^{* *} \mathrm{P}<0.01$. ${ }^{\dagger}$ Adjusted for age, menarche status, height, weight, serum 25-OH vitamin D, physical activity (METs · min/wk), energy intake, and calcium intake using linear regression analysis with complex sampling design. ${ }^{\ddagger}$ Adjusted for age, menarche status, height, bone mass-free lean mass of whole body, serum 25-OH vitamin D, physical activity (METs · min/wk), energy intake, and calcium intake using linear regression analysis with complex sampling design. 
However, the associations were not more significant when bone mass-free lean mass of whole body was substituted for body weight as a confounder regardless of gender. On the other hand, the associations between TEFR and BMD at whole body and regional BMD were gender-specific: for girls, the relationships were inverse associations in the body weight adjusted models but were not significant in the bone mass-free lean mass of whole body adjusted models; for boys, the associations were positive regardless of adjustment for body weight or bone mass-free lean mass of whole body.

Regional body fat mass, especially abdominal body fat mass, was related with BMD in previous studies. Magnetic resonance imaging-measured abdominal visceral fat had an inverse relationship with spinal and whole BMD of obese or non-obese 30 female adolescents. ${ }^{14)}$ In the study about 175 obese adolescents, after adjusting for whole and abdominal body fat mass, abdominal fat thickness which measured by ultrasonography and BMD had negative relationships. ${ }^{13)}$ The pathophysiology of inverse association between abdominal fat and BMD has been explained by the unfavorable effects of adipokine from abdominal fat on bone growth-related factors or insulin resistance associated with visceral fat. ${ }^{14,20)}$ As compared with these two studies, we examined the association between trunk fat that covers chest and pelvic fat as well as abdominal fat using a different measurement method and adjustment with different confounding variables in obese and no-obese adolescents. In this study, the strength of associations between body fat percent and BMD were attenuated after adjusting for bone mass-free lean mass of whole body. This finding suggests that the apparent associations between body fat percent and $\mathrm{BMD}$ could be due to bone mass contained in body weight.

The underlying mechanisms for gender-specific associations between TEFR and BMD after adjusting for bone mass-free lean mass of whole body is unclear. The differences in sex hormone such as the effects of estrogen on body fat ${ }^{21,22)}$ and cortical and trabecular bone ${ }^{23)}$ in girls may play a role in the genderdifferences. Although the pathophysiology should be clarified, these findings suggest that increase in fat mass of trunk relative to extremities may favorable for increase in BMD in boys regardless of bone mass-free lean body mass.

There are several limitations need to be considered. First, there may be measurement errors according to subjects' age, height, weight, BMD, and body composition ${ }^{24)}$ although DEXA is a standard method of BMD measurement. Second, uncontrolled residual confounding variables like sexual development and disease status could influence on the relationships. Third, the study design is cross-sectional study and then, a causal relationship between body composition and BMD cannot be assessed. Lastly, these findings could not infer the relationship between body fat and BMD in other regions. In spite of these limitations, our results may be generalized to Korean adolescents since the data represented the adolescent populations in Korea.

In conclusion, after adjusting for bone mass-free lean mass of whole body, body fat percent were not independently related to BMD in adolescents, while ratio of trunk fat mass to extremities fat mass was positively associated with BMD in boys but not in girls.

\section{CONFLICT OF INTEREST}

No potential conflict of interest relevant to this article was reported.

\section{REFERENCES}

1. Ray NF, Chan JK, Thamer M, Melton LJ 3rd. Medical expenditures for the treatment of osteoporotic fractures in the United States in 1995: report from the National Osteoporosis Foundation. J Bone Miner Res 1997;12:24-35.

2. NIH Consensus Development Panel on Osteoporosis Prevention, Diagnosis, and Therapy. Osteoporosis prevention, diagnosis, and therapy. JAMA 2001;285:785-95.

3. Jin MR, Kim JM, Kim H, Chang N. Associations of lifestyle behaviors, dietary habits and bone mineral density in men aged 50 years and older. Korean J Nutr 2009;42:59-67.

4. Bates DW, Black DM, Cummings SR. Clinical use of bone densitometry: clinical applications. JAMA 2002;288:1898900.

5. Nelson HD, Haney EM, Dana T, Bougatsos C, Chou R. Screening for osteoporosis: an update for the U.S. Preventive Services Task Force. Ann Intern Med 2010;153:99-111.

6. Reid IR, Plank LD, Evans MC. Fat mass is an important 
determinant of whole body bone density in premenopausal women but not in men. J Clin Endocrinol Metab 1992;75:779-82.

7. Reid IR. Relationships between fat and bone. Osteoporos Int 2008;19:595-606.

8. Cui LH, Shin MH, Kweon SS, Park KS, Lee YH, Chung EK, et al. Relative contribution of body composition to bone mineral density at different sites in men and women of South Korea. J Bone Miner Metab 2007;25:165-71.

9. Makovey J, Naganathan V, Sambrook P. Gender differences in relationships between body composition components, their distribution and bone mineral density: a cross-sectional opposite sex twin study. Osteoporos Int 2005; 16:1495-505.

10. Gjesdal CG, Halse JI, Eide GE, Brun JG, Tell GS. Impact of lean mass and fat mass on bone mineral density: The Hordaland Health Study. Maturitas 2008;59:191-200.

11. Pietrobelli A, Faith MS, Wang J, Brambilla P, Chiumello G, Heymsfield SB. Association of lean tissue and fat mass with bone mineral content in children and adolescents. Obes Res 2002;10:56-60.

12. El Hage RP, Courteix D, Benhamou CL, Jacob C, Jaffre C. Relative importance of lean and fat mass on bone mineral density in a group of adolescent girls and boys. Eur J Appl Physiol 2009; 105:759-64.

13. Junior IF, Cardoso JR, Christofaro DG, Codogno JS, de Moraes AC, Fernandes RA. The relationship between visceral fat thickness and bone mineral density in sedentary obese children and adolescents. BMC Pediatr 2013;13:37.

14. Russell M, Mendes N, Miller KK, Rosen CJ, Lee H, Klibanski A, et al. Visceral fat is a negative predictor of bone density measures in obese adolescent girls. J Clin Endocrinol Metab 2010;95:1247-55.

15. Lee K. Regional percent fat and bone mineral density in Korean adolescents: the Fourth Korea National Health and Nutrition Examination Survey (KNHANES IV-3), 2009.
Asia Pac J Clin Nutr 2013;22:69-73.

16. Korea Centers for Disease Control and Prevention. Guidelines for using the Fifth National Health and Nutrition Examination Survey (KNHANES V-1), 2010. Cheongju: Korea Centers for Disease Control and Prevention; 2011.

17. Korea Centers for Disease Control and Prevention. Education and quality control of bone mineral density in the Fourth National Health and Nutrition Examination Survey (KNHANES IV-3), 2009. Cheongju: Korea Centers for Disease Control and Prevention; 2010.

18. IPAQ Research Committee. Guidelines for data processing and analysis of the International Physical Activity Questionnaire (IPAQ): short and long forms [Internet]. Stockholm: Karolinska Institutet; 2005 [cited 2012 Mar 22]. Available from: http://www.ipaq.ki.se/scoring.pdf.

19. Consensus development conference: diagnosis, prophylaxis, and treatment of osteoporosis. Am J Med 1993;94:646-50.

20. Campos RM, Lazaretti-Castro M, Mello MT, Tock L, Silva PL, Corgosinho FC, et al. Influence of visceral and subcutaneous fat in bone mineral density of obese adolescents. Arq Bras Endocrinol Metabol 2012;56:12-8.

21. Afghani A, Goran MI. The interrelationships between abdominal adiposity, leptin and bone mineral content in overweight Latino children. Horm Res 2009;72:82-7.

22. Afghani A, Goran MI. Racial differences in the association of subcutaneous and visceral fat on bone mineral content in prepubertal children. Calcif Tissue Int 2006;79:383-8.

23. Hong X, Arguelles LM, Tsai HJ, Zhang S, Wang G, Wang B, et al. Plasma adipokines, bone mass, and hip geometry in rural Chinese adolescents. J Clin Endocrinol Metab 2010;95:164452.

24. Laskey MA, Lyttle KD, Flaxman ME, Barber RW. The influence of tissue depth and composition on the performance of the Lunar dual-energy X-ray absorptiometer whole-body scanning mode. Eur J Clin Nutr 1992;46:39-45. 